\title{
Image-Based Brachytherapy in Cervical Cancer: Review and Experiences in Faculty of Medicine, Chiang Mai University
}

\author{
Ekkasit Tharavichitkul*, Somvilai Chakrabandhu, Pitchayaponne Klunklin, Wimrak Onchan, \\ Imjai Chitapanarux
}

The Division of Therapeutic Radiology and Oncology, Department of Radiology, Faculty of Medicine, Chiang Mai University, Chiang Mai, Thailand.

Email: "paan_31@hotmail.com

Received March 1 ${ }^{\text {st }}, 2013$; revised April 1 ${ }^{\text {st }}, 2013$; accepted April 10 $0^{\text {th }}, 2013$

Copyright (C) 2013 Ekkasit Tharavichitkul et al. This is an open access article distributed under the Creative Commons Attribution License, which permits unrestricted use, distribution, and reproduction in any medium, provided the original work is properly cited.

\begin{abstract}
Cervical cancer is the one of the most common cancer in female patients in Thailand. Radiotherapy has the role for the treatment of cervical cancer by postoperative, radical and palliative treatments. For radical radiotherapy, the combination of external beam radiation therapy and brachytherapy will be used to increase the tumor dose to curative goal. With the new development of medical images (Computed tomography (CT), Magnetic Resonance Imaging (MRI) or Ultrasonography (US)), the treatment with brachytherapy will be developed from point-based to volume-based concepts. Many studies reported the benefit of image-based brachytherapy over conventional brachytherapy and clinical benefit of using image-based brachytherapy in the treatment of cervical cancer.
\end{abstract}

Keywords: Image-Based Brachytherapy; Cervical Cancer; Review

\section{Role of Radiotherapy for Cervical Cancer}

Cervical cancer is one of the most common in female populations in Thailand. From our institution, the numbers of cervical carcinoma were 234 patients in the year of 2005 [1]. Treatment of cervical cancer composed of surgery, radiation therapy and systemic treatments. The roles of radiation therapy in cervical cancer are radical, adjuvant and palliative treatments. In early stages, surgery plays the main role of treatment. Radiotherapy can be used as adjuvant to reduce the risks of recurrence. However, in some patients who are unfit to perform surgery, radiotherapy is an option. In advanced disease, concurrent chemoradiation with cisplatin-based regimen is the standard treatment. Many studies showed the benefit of concurrent chemoradiation over radiotherapy alone [2-4]. For radiotherapeutic treatment, external beam radiation (EBRT) and brachytherapy are used together to increase the dose to tumor at cervix and reduce the dose to organs at risk (bladder and rectum).

\footnotetext{
${ }^{*}$ Corresponding author.
}

\section{Intracavitary Brachytherapy for Cervical Cancer}

Brachytherapy was used to increase the curative dose to cervical cancer from the year of when Todd and Meredith et al. introduced the Manchester system with radium [5]. The using of point $\mathrm{A}$, bladder point and rectum point (identified in orthogonal X-rays) were widespread with ICRU 38 concepts. The intrauterine tandem and vaginal colpostats or ring applicator were used in many institutes to treat cervical cancer, with acceptable results. Figure 1 shows orthogonal X-rays for conventional planning.

\section{Image-Based Brachytherapy for Cervical Cancer}

However, the using of film-based planning have still some problems due to could not evaluate the real anatomy of target lesion and multiple organs at risk. With the emerging of imaging technologies (Computed tomography, Magnetic resonance imaging and ultrasound), the question of imaging will helps to optimize the treatment 


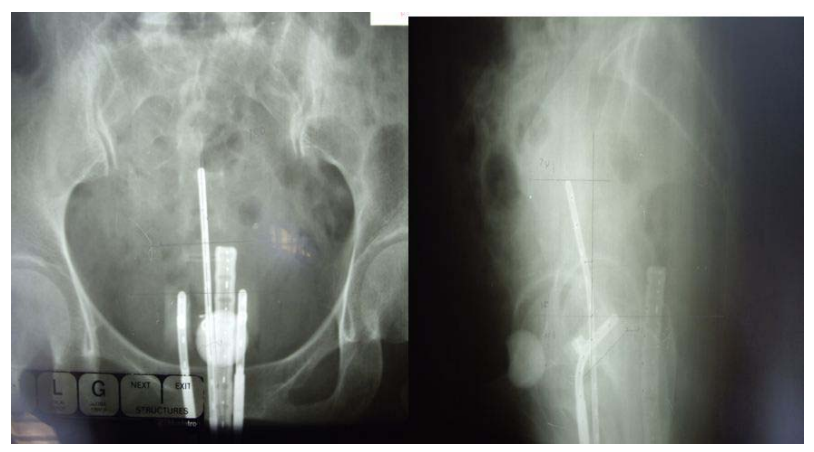

Figure 1. Orthogonal X-rays of intracavitary brachytherapy for cervical carcinoma.

and the concepts of prescription will be developed from point-based planning (point A) to volume-based planning. Nowadays, studies of developments are published.

\subsection{Volume-Based Concepts in Brachytherapy for Cervical Cancer}

With the limitation of X-ray based planning, the concepts of volume-based planning were evaluated. In the year of 2005-2006, The GEC-ESTRO recommendations were published. The volume-based concepts of target volume and organs at risk (OARs) with the dose-volume histograms for each target were identified in magnetic resonance imaging. For target volumes, these composed Gross tumor volume (GTV), High-risk clinical target volume (HR-CTV) and Intermediate-risk Clinical target volume (IR-CTV). HR-CTV was defined to GTV plus the whole cervix with the suspected extension describing on magnetic resonance imaging that represents the tumor at brachytherapy ( $85-90 \mathrm{~Gy}$ ). IR-CTV was identified to HR-CTV plus $5-15 \mathrm{~mm}$ margin excluded the OARs which relate to tumor at diagnosis (60 Gy). For the OARs, bladder, rectum and sigmoid colon were identified. For the dose constraint, the D90 (dose at $90 \%$ of volume), D100 (dose at $100 \%$ of volume) were used to determine to the tumor dose. The D2 cc (dose at $2 \mathrm{cc}$ of volume), D1cc and D0.1cc were used as OARs dose limitation. For the recommendations, The D90 to HR-CTV should be more than $85-90$ Gy in locally advanced cervical cancer while the D2 cc for bladder, rectum and sigmoid were 90 - $95 \mathrm{~Gy}, 70$ - 75 Gy and 70 - 75 Gy, respectively $[6,7]$.

\subsection{Comparison of Standard Loading versus Image-Guided Planning}

Many studies supported the using of image-guided brachytherapy (IGBT) to improve dose distribution in treatment of cervical cancer. De Brabandere et al. reported 16 patients who were boosted with PDR brachytherapy after EBRT. X-ray based plan were projected to
MRI and GTV, HR-CTV, IR-CTV were retrospectively contoured and OARs (bladder, rectum and sigmoid colon). Dose-Volume-histograms (DVHs) were evaluated and second phase optimization was performed to lower the dose in OARs (bladder $<85 \mathrm{~Gy}$, rectum and sigmoid $<75$ Gy, HR-CTV > 85 Gy). The X-ray based plan yielded the D2 cc for bladder and rectum exceeded the tolerance doses in 10/16 and 7/16 patients, respectively and IR-CTV coverage at $60 \mathrm{~Gy}$ was acceptable. D90 of HR-CTV was lower than 85 Gy in 13/16. After optimization, no overdose was found. The average dose decrease in OARs: bladder $7+/-6$ Gy, sigmoid $7+/-4$ Gy and average dose increase in HR-CTV in 3 Gy [8]. Vinod et al. reported 20 patients with $62 \mathrm{CT}$ datasets treated with definitive RT (EBRT 45 Gy plus BT (CT-or MRI-based planning) $24 \mathrm{~Gy} / 3$ fractions). The D2 cc versus ICRU point dose were 4.28 versus $4.01 \mathrm{~Gy}=1.08$ in rectum, Bladder: 8.65 vs. $6.74 \mathrm{~Gy}=1.39$ in bladder and $4.58 \mathrm{~Gy}$ in sigmoid (no sigmoid dose in ICRU point dose), respectively. The Correlation of DICRU/D2 cc rectum was $0.76(\mathrm{p}=0.001)$ and the Correlation of DICRU/D2 cc bladder was $0.78(\mathrm{p}=0.01)$ [9]. Tanderup et al. published 72 consecutive cervical cancer patients performed MRI-based IGABT with GEC-ESTRO recommendations. The point doses were compared to $3 \mathrm{D}$ dose parameters for tumor and OARs. HR-CTV D90 highly variable in standard plan with point A. In small tumor (volume $<31$ cc), HRCTV was well covered by standard plan $94 \%$ while OARs exceeded in $72 \%$. With optimization, the OARs overdose could be reduced to $6 \%$ and maintain excellent target coverage. In large tumor (volume $>31$ cc), HRCTV was covered by standard plan $25 \%$. Optimization resulted in full coverage in $72 \%$ and reduced the exceed dose to OARs [10]. Wanderås et al. reported brachytherapy data with Fletcher applicator from 19 patients with a total of 72 individual treatment fractions with CT-based planning. The doses of standard plan versus optimized plan were retrospectively reviewed. For standard treatment planning, the tolerance dose limits were exceeded in the bladder, rectum and sigmoid in $26 \%, 4 \%$ and $15 \%$ of the plans, respectively. An increase in target dose coverage was achieved when additional individual optimization was performed, while still keeping the dose to the OARs below predefined limits. Relatively low average target coverage, especially for the largest volumes was however seen [11]. Zwahlen et al. reported 20 patients with IB-IV by FIGO cervical cancer designed for IGABT-PDR with MRI-guided treatment planning. HR-CTV and OARs were identified according to GEC-ESTRO recommendations and Optimization was performed and compared with the conventional plan. The Conventional plan adequately treated $70 \%$ for the HR-CTV and IR-CTV $85 \%$ for the IR-CTV. With optimization, $75 \%$ for HR-CTV could adequately treat while 
95\% for IR-CTV could be covered. MRI-guided BT could reduce the dose to OARs $(12 \%-32 \%)$ when compared to conventional BT planning [12]. Tharavichitkul et al. reported using of CT-based brachytherapy for 17 patients with cervical cancer. All patients were treated with $46-50$ Gy of external beam radiotherapy and at least 7 Gy $\times 4$ fractions to the D90 of HR-CTV. CT-based brachytherapy significantly reduced the dose at D2 cc of bladder and rectum when compared with standard planning (to point A) [13]. Moreover, the study from Faculty of medicine, Chiang Mai University reported using of MRI-guided brachytherapy in 15 patients with locally advanced cervical cancer. All patients were treated with $46-50$ Gy of external beam radiotherapy and at least $7 \mathrm{~Gy} \times 4$ fractions to the D90 of HR-CTV. Three times of MRI were designed at diagnosis, first application of brachytherapy and three months after treatment finished. In comparison to conventional approach (point A basic), MRI-guided adaptive brachytherapy could reduce the dose of all of Organs at risk significantly and the dose to HR-CTV could be kept more than 87 Gy EQD2 according to Vienna study [14]. Nowadays, many studies showed that some of patients have improperly anatomical geometries for standard loading pattern causing suboptimal dose to tumor and overdose to organs at risk. From the data of imageguided treatment, IGBT helps improve the dose distribution to target volumes and organs at risk.

\subsection{MRI-Guided Brachytherapy for Cervical Cancer}

With the benefit of tissue discrimination form MRI, high quality of image in multi-planar aspects and provides clearly tissue discrimination of Target (tumor) extension and OARs. Figure 2 illustrates MRI image showing GTV, HR-CTV and OARs. Nowadays, the clinical supports were published. Pötter et al. reported 156 patients with locally advanced cervical cancer treated with 45 50 Gy EBRT plus $7 \times 4$ Gy in HR-CTV according to GEC-ESTRO recommendations. The mean D90 of HR-CTV was 93 Gy while the mean D2 cc for urinary bladder, rectum and sigmoid colon were $86 \mathrm{~Gy}, 65 \mathrm{~Gy}$ and $64 \mathrm{~Gy}$, respectively. The overall 3-yr local control was $95 \%(2-5 \mathrm{~cm} 98 \%,>5 \mathrm{~cm} 92 \%$ and by stage: IB $100 \%$, IIB 96\%, IIIB 86\%). The overall Cancer specific survival rate at 3 yr was $74 \%(2-5 \mathrm{~cm} 83 \%,>5 \mathrm{~cm} 70 \%$ and by stage: IB $83 \%$, IIB $84 \%$, IIIB $52 \%$ ). The Overall survival rate at 3 yr was $68 \%(2-5 \mathrm{~cm} 72 \%,>5 \mathrm{~cm} 65 \%$ and by stage: IB 74\%, IIB 78\%, IIIB 45\%). The total Late morbidity for G1-2 vs. G3-4 were 188 events vs. 11 events (Bladder: G1-2/G3-4 = 32/3, Rectum: 14/5, Sigmoid: 3/0, Vagina: 128/2) [15]. Dimopoulos et al. reported 141 patients with cervical cancer (stage IB-IVA)

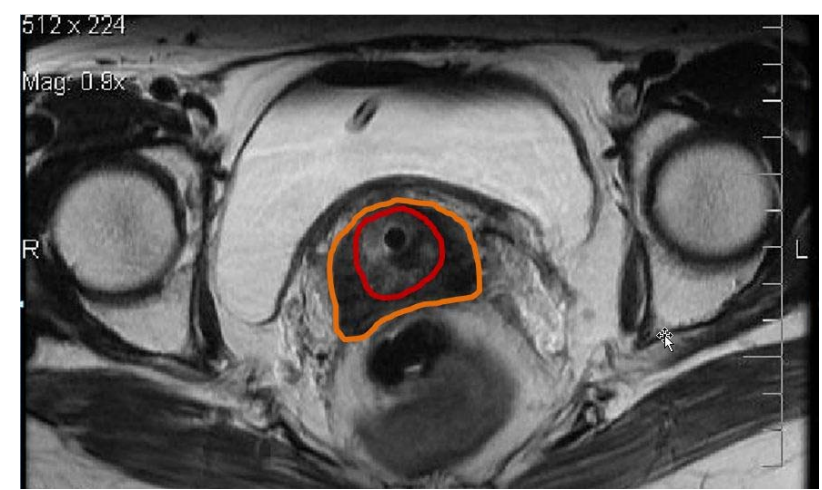

Figure 2. MRI-guided brachytherapy showed the better soft tissue discrimination of gross tumor volume $(\mathrm{GTV}=$ red color), clinical target volume $(\mathrm{CTV}=$ orange color $)$ and organs at risk.

treated with 45 - 50 Gy EBRT plus $7 \times 4$ Gy in HR-CTV according to GEC-ESTRO recommendations. For the study, eighteen local recurrences occurred. The most important DVH parameters correlated with LR were the D90 and D100 for HR-CTV mean D90 and D100 values for HRCTV were $86+/-16$ and $65+/-10 \mathrm{~Gy}$.

The D90 $>87$ Gy had local recurrence rate of $4 \%$ while D90 $<87$ Gy had local recurrence rate of $20 \%$. The dose related to local control, D90 > 87 Gy yield high local control [16]. Moreover, George et al. published the $\mathrm{D} 2 \mathrm{cc}$ and D1 $\mathrm{cc}$ as good predictive factor for rectal toxicity [17].

\subsection{CT- or MRI-Guided Brachytherapy for Cervical Cancer}

CT the good tissue discrimination of Organs at risks but the identification of tumor was less than MRI. Figure 3 illustrates the CT image with HR-CTV and OARs. In advantage point, most of radiation oncology units nowadays have $\mathrm{CT}$ in the Unit that means high assesment. Although it is more convenient, it has less real-time benefit than ultrasound. Many studies compared the CT to MRI images. Viswanathan et al. reported 10 patients performed both MRI and CT after applicator insertion. Tumor height, thickness, and total volume measurements, as determined by either CT or CT (Std) were not signifycantly different compared with the MRI volumes. In contrast, the width measurements differed in HR-CTV $(\mathrm{CTStd})(\mathrm{p}=0.05)$ and IR-CTV $(\mathrm{CTStd})(\mathrm{p}=0.01)$. For the HR-CTV (CTStd), this resulted in statistically significant differences in the volume treated to the prescripttion dose or greater (MRI, 96\% vs. CT (Std), 86\%, p = 0.01), D (100) (MRI, 5.4 vs. CT (Std), 3.4, p < 0.01), and D (90) (MRI, 8.7 vs. CT (Std), 6.7, p < 0.01). Correspondingly, the IR-CTV DVH values on MRI vs. CT (Std), differed in the D (100) (MRI, 3.0 vs. CT (Std), 2.2, $\mathrm{p}=0.01)$ and D (90) (MRI, 5.6 vs. CT (Std), 4.6, p = 


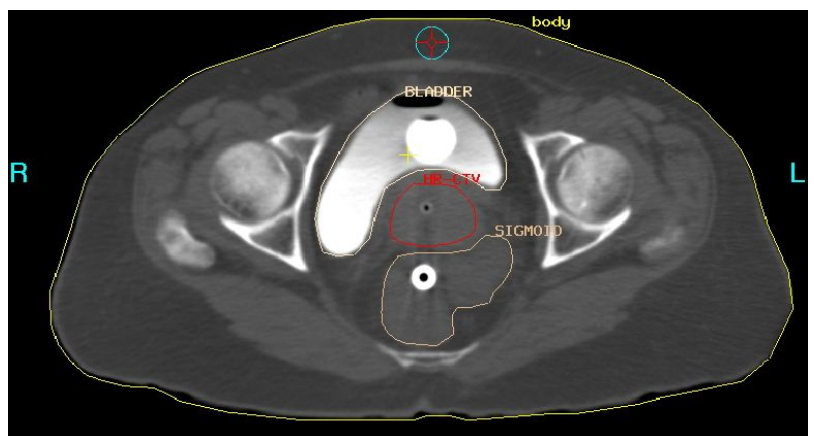

Figure 3. CT-based intracavitary brachytherapy in cervical cancer which showed the HR-CTV (red), urinary bladder and sigmoid colon.

0.02). The MRI and CT DVH values of the dose to 0.1 $\mathrm{cm}^{3}, 1 \mathrm{~cm}^{3}$, and $2 \mathrm{~cm}^{3}$ for the OARs were similar. They concluded that computed tomography-based or MRIbased scans at brachytherapy are adequate for OAR DVH analysis. However, CT tumor contours can significantly overestimate the tumor width, resulting in significant differences in the D (90), D (100), and volume treated to the prescription dose or greater for the HR-CTV compared with that using MRI [18]. Eskander et al. reported the comparison of CT versus MRI on 11 patients. Tandem and ring applicator were used to all patients. The radiation oncologist identified and contoured the rectum, the bladder, the sigmoid, and the high-risk clinical target volume (HR-CTV) on CT and MRI. The values compared between CT and MRI included D90 and D100 to HR-CTV; coronal, sagittal, and axial measurements of HR-CTV; and minimum dose to most irradiated 0.1-, $0.5-, 1.0-$, and $2.0-\mathrm{cm}^{3}$ volumes for the organs at risk (OAR). Doses were converted to the equivalent dose in 2 Gy by applying the linear quadratic model. Volume optimization was performed, and the above parameters were evaluated. Magnetic resonance imaging showed a significantly greater HR-CTV length in the sagittal plane $(p=0.006)$, with CT showing a greater length in the coronal plane $(p=0.004)$. The equivalent dose in $2 \mathrm{~Gy}$ to $2.0 \mathrm{~cm}^{3}$ for the bladder was greater on CT than MRI $(\mathrm{p}=$ 0.041 ). The dose volume histogram values for the other OARs were similar between CT and MRI. With volume optimization, no significant differences were seen between HR-CTV dose parameters or doses to OAR. They concluded that.

The CT- and MRI-based brachytherapy tissue delineation seems adequate for evaluation of OAR and target tissues. Although the shapes of HR-CTV and OAR do differ, the clinical impact of these differences seems to be small and may demonstrate that planning with CT, if combined with one MRI, may be sufficient [19]. Krishnatry et al. published 17 previously untreated patients with cervical cancer. Brachytherapy using a MRI-com- patible applicator followed by both CT and MRI were applied and compared. The HR-CTV and organs at risk (bladder, rectum, sigmoid and intestines) were contoured on CT using only clinical findings and on MRI using GEC-ESTRO guidelines. The volume and doses for tumor and organs at risk were evaluated using two-sided t-test. There is significant underestimation of tumor height and overestimation of the width $(p<0.05)$. However, there was no significant difference in V (100), D (90) and D (100) for high- and intermediate-risk clinical target volume in computed tomography versus magnetic resonance imaging. The volumes and doses to $0.1,1$ and $2 \mathrm{cc}$ for organs at risk were also similar. They concluded that MRI is the gold standard for tumor delineation, but CT-based contouring can be used comfortably for delineation of organs at risk [20]. From all literatures, CT can be used to delineate organs at risk to adjust the critical doses to cause toxicity. Although, the image identification of GTV by CT is poorer than MRI, Hybrid CT plus MRI guidance helps to identify the gross tumor volume during brachytherapy but $\mathrm{CT}$ can be acceptable to use image-based brachytherapy. The clinical impact of CT-based brachytherapy was reported by Tan et al. with twenty-eight patients. All patients received EBRT followed by HDR brachytherapy $(7 * 3)$ with CT-guided BT. Cervix, uterus and OARs were contoured according to GEC-ESTRO recommendations. For target, D90 and V100 were evaluated and for OARs, D2 cc were evaluated by EQD2. The 3-year cancer-specific survival was $81 \%$, with a pelvic control rate of $96 \%$. In 24 pts, the D90 equal to $74 \mathrm{~Gy}$ or more (alpha/beta $=10$ ). The patients who had local recurrence had D90 of $63.8 \mathrm{~Gy}$. Serious late morbidity was $14 \%$. Seventeen patients had satisfactory OAR doses by standard loading pattern and 7 patients had to optimize to reduce risk of toxicity [21].

\subsection{Ultrasound-Guided Brachytherapy for Cervical Cancer}

In comparison to other images, ultrasound shows less tissue discrimination for identification of target and Organs at risk but the benefit of ultrasound is that it is the only one real time imaging for the treatment process. Figure 4 shows the ultrasound image that illustrates the correlation between tandem and uterus. Van Dyk et al. reported 71 patients with locoregionally advanced cervix cancer treated with chemoradiation and brachytherapy. US-assisted tandem insertion and conformal US-based planning were used. Orthogonal films for applicator reconstruction were also taken. A standard plan (STD) was modified to suit the US-based volume and treatment was delivered. The patient then underwent a magnetic resonance imaging (MRI) scan with the applicators in situ. Standard plans had higher doses to point A, target vol- 


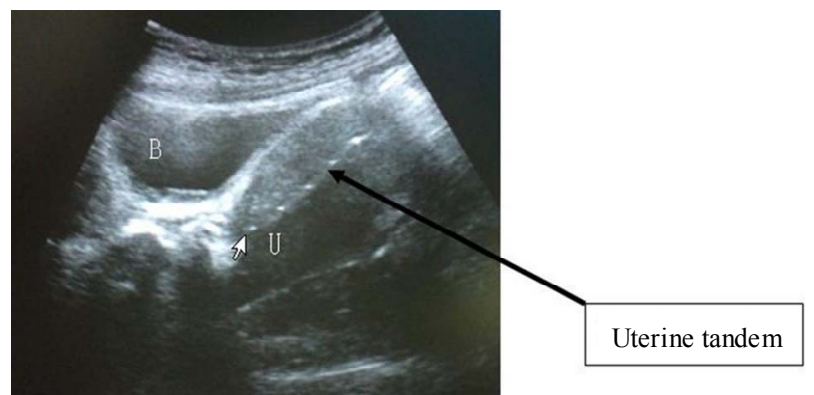

Figure 4. The ultrasound image in sagittal view shows the bladder, cervix, uterus and uterine tandem (arrow); the correlation of uterine tandem and anterior/posterior borders of uterus were evaluated.

ume, ICRU 38 bladder and rectal points, and individualized bowel point compared with US and MRI plans. There was a statistically significant difference between standard plans and image-based plans, STD vs. US, STD vs. MRI, and STD vs. Final-having consistent ( $\mathrm{p}<$ or $=$ 0.001 ) respectively for target volume, Point A, ICRU 38 bladder, and bowel point. US plan assessed on two-dimensional MRI image was comparable for target volume $(\mathrm{p}=0.11)$, rectal point $(\mathrm{p}=0.8)$, and vaginal mucosa $(\mathrm{p}=$ 0.19 ). The local control was $90 \%$ and late bowel morbidity (G3, G4) was <2\% [22]. Mahantshetty et al. published the using of transrectal ultrasound correlated to the magnetic resonance imaging in cervical cancer for 20 patients. The D1 and D3 (anterior surface) had strong correlation between TRUS and MRI R $=0.92$ and $0.94(\mathrm{p}$ $<0.01$ ) and D2 and D4 reference (posterior surface) had moderate correlation between them with $\mathrm{R}=0.62$ and $0.82(p<0.01)$. The D5 reference (Fundal thickness) had strong correlation between TRUS and US with $\mathrm{R}=0.98$. The D2 references showed the least correlation between the two images [23]. From the literatures, US-based planning was comparable to CT or MRI.

\subsection{The Improvement of Planning: Inversed Planning Brachytherapy}

The using of inversed planning in brachytherapy was started in research. In gynecologic brachytherapy, inverse planning was studied in the two main algorithms. The first algorithm is IPSA (inverse planning systematic annealing) and another one is the Hybrid inverse treatment planning and optimization (HIPO). Trnkova et al. reported the using of HIPO algorithm for image-guided brachytherapy versus conventional manual optimized IGBT. Ten cases of intracavitary brachytherapy (IC) and 10 cases of intracavitary/interstitial brachytherapy (IC/IS) were evaluated. The results showed that HIPO could achieve a better target coverage (V100) for all IC and 7 IC/IS patients. The $\mathrm{D} 2 \mathrm{cc}$ per fraction for bladder, rectum and sigmoid colon were an average lower by $0.2 \mathrm{~Gy}, 0.4$
Gy, 0.2 Gy by IC and 0.6 Gy, 0.3 Gy, 0.3 Gy by IC/IS, respectively. The sparing factors were better for HIPO plans. Additionally, the D2 cc, D1 cc and D0.1 cc for vaginal wall were lover and smaller area in HIPO [24]. Chajon et al. reported Inverse planning approach for three-dimensional IGBT-PDR in 30 patients with customized vaginal mold. Dose-volume-histogram (DVH) obtained using Inverse Planning Simulated annealing (IPSA) software for the optimization of dose distribution versus manual optimization method (MOM). Target volumes/OARs were delineated according to GEC-ESTRO recommendations. The mean D90, D10 and V100 with both methods did not differ significantly in Bladder ICRU point: 58.4 Gy for MOM vs. 55 Gy for IPSA ( $\mathrm{p}=$ 0.0001). For Rectum ICRU point, IPSA lowered the dose in comparison to MOM [25]. Kim et al. published 51 patients with FIGO IB1-IVA cervical cancer treated by IPSA-planned HDR BT boost to the EQD2 dose $=85 \mathrm{~Gy}$ or greater (in alpha/beta $=10)$. At median follow-up time of 24.3 months, grade 3 toxicities were found in $4 \%$ for acute and $2 \%$ for late effects, respectively. No Grade 4 toxicity was observed. For Acute G1-2 toxicity, there were $48 \%$ for GI and $52 \%$ for GU toxicity, respectively. Thirty-one percents developed late G1-2 toxicity. Two patients developed local recurrence; 2 for regional recurrences and 15 for distant metastasis. The 2-year locoregional control was $91 \%$ with 2-years OS of $86 \%$ [26].

\section{Conclusion}

The developments of brachytherapy shift from conventional approach to be high-technologically image-based process. The volume-based concepts and dose-volumehistograms were used to improve the quality of treatments by brachytherapy with increasing the dose to target while reducing the dose to organs at risks.

\section{Acknowledgements}

The author offers many thanks to the staff of the NRUCMU in the Gynecologic Oncology Cluster and the Division of Therapeutic Radiology and Oncology, Faculty of Medicine, Chiang Mai University for supporting this study.

\section{REFERENCES}

[1] P. Kamnerdsupaphon, S. Srisukho, Y. Sumitsawan, V. Lorvidhaya and V. Sukthomya, "Cancers in Northern Thailand," Biomedical Imaging and Intervention Journal, Vol. 4, No. 3, 2008, p. e46. doi:10.2349/biij.4.3.e46

[2] P. G. Rose, B. N. Bundy, E. B. Watkins, J. T. Thigpen, G. Deppe, M. A. Maiman, et al., "Concurrent CisplatinBased Radiotherapy and Chemotherapy for Locally Advanced Cervical Cancer," New England Journal of Medi- 
cine, Vol. 340, No. 15, 1999, pp. 1144-1153. doi:10.1056/NEJM199904153401502

[3] H. M. Keys, B. N. Bundy, F. B. Stehman, L. I. Muderspach, W. E. Chafe, C. L. Suggs 3rd, et al., "Cisplatin, Radiation, and Adjuvant Hysterectomy Compared with Radiation and Adjuvant Hysterectomy for Bulky Stage IB Cervical Carcinoma," New England Journal of Medicine, Vol. 340, No. 15, 1999, pp. 1154-1161. doi:10.1056/NEJM199904153401503

[4] P. J. Eifel, K. Winter, M. Morris, C. Levenback, P. W. Grigsby, J. Cooper, et al., "Pelvic Irradiation with Concurrent Chemotherapy versus Pelvic and Para-aortic Irradiation for High-Risk Cervical Cancer: An Update of Radiation Therapy Oncology Group Trial (RTOG) 90-01," Journal of Clinical Oncology, Vol. 22, No. 5, 2004, pp. 872-880. doi:10.1200/JCO.2004.07.197

[5] M. Tod and W. J. Meredith, "Treatment of Cancer of the Cervix Uteri, a Revised Manchester Method," British Journal of Radiology, Vol. 26, No. 305, 1953, pp. 252-257. doi:10.1259/0007-1285-26-305-252

[6] C. Haie-Meder, R. Ptter, E. Van Limbergen, I. Barillot, M. De Brabandere, J. Dimopoulos, et al., "Recommendations from Gynaecological (GYN) GEC-ESTRO Working Group (I): Concepts and Terms in 3D Image Based 3D Treatment Planning in Cervix Cancer Brachytherapy with Emphasis on MRI Assessment of GTV and CTV," Radiotherapy \& Oncology, Vol. 74, No. 3, 2005, pp. 235245. doi:10.1016/i.radonc.2004.12.015

[7] R. Pötter, C. Haie-Meder, E. Van Limbergen, I. Barillot, M. De Brabandere, J. Dimopoulos, et al., "GEC ESTRO Working Group. Recommendations from Gynecological (GYN) GEC ESTRO Working Group (II): Concepts and Terms in 3D Image-Based Treatment Planning in Cervix Cancer Brachytherapy-3D Dose Volume Parameters and Aspects of 3D Image-Based Anatomy, Radiation Physics, Radiobiology," Radiotherapy \& Oncology, Vol. 78, No. 1, 2006, pp. 67-77. doi:10.1016/j.radonc.2005.11.014

[8] M. De Brabandere, A. G. Mousa, A. Nulens, A. Swinnen and E. Van Limbergen, "Potential of Dose Optimisation in Mri-Based PDR Brachytherapy of Cervix Carcinoma," Radiotherapy \& Oncology, Vol. 88, No. 2, 2008, pp. 217226. doi:10.1016/i.radonc.2007.10.026

[9] S. K. Vinod, K. Caldwell, A. Lau and A. R. Fowler, "A Comparison of ICRU Point Doses and Volumetric Doses of Organs at Risk (OARs) in Brachytherapy for Cervical Cancer," Journal of Medical Imaging and Radiation Oncology, Vol. 55, No. 3, 2011, pp. 304-310. doi:10.1111/j.1754-9485.2011.02272.x

[10] K. Tanderup, S. K. Nielsen, G. B. Nyvang, E. M. Pedersen, L. Røhl, T. Aagaard, et al., "From Point A to the Sculpted Pear: MR Image Guidance Significantly Improves Tumour Dose and Sparing of Organs at Risk in Brachytherapy of Cervical Cancer," Radiotherapy \& Oncology, Vol. 94, No. 2, 2010, pp. 173-180. doi:10.1016/j.radonc.2010.01.001

[11] A. D. Wanderås, M. Sundset, I. Langdal, S. Danielsen, G. Frykholm and A. B. Marthinsen, "Adaptive Brachytherapy of Cervical Cancer, Comparison of Conventional Point A and CT Based Individual Treatment Planning," Acta
Oncologica, Vol. 51, No. 3, 2012, pp. 345-354. doi:10.3109/0284186X.2011.626446

[12] D. Zwahlen, J. Jezioranski, P. Chan, M. A. Haider, Y. B. Cho, I. Yeung, et al., "Magnetic Resonance ImagingGuided Intracavitary Brachytherapy for Cancer of the Cervix," International Journal of Radiation Oncology Biology Physics, Vol. 74, No. 4, 2009, pp. 1157-1164. doi:10.1016/j.ijrobp.2008.09.010

[13] E. Tharavichitkul, S. Mayurasakorn, V. Lorvidhaya, V. Sukthomya, S. Wanwilairat, S. Lookaew, et al., "Preliminary Results of Conformal Computed Tomography (CT)-based Intracavitary Brachytherapy (ICBT) for Locally Advanced Cervical Cancer: A Single Institution's Experience," Journal of Radiation Research, Vol. 52, No. 5, 2011, pp. 634-640. doi:10.1269/jrr.10154

[14] E. Tharavichitkul, C. Sivasomboon, S. Wanwilairat, V. Lorvidhaya, V. Sukthomya, S. Chakrabhandu, et al., "Preliminary Results of MRI-Guided Brachytherapy in Cervical Carcinoma: The Chiangmai University Experience," Journal of Radiation Research, Vol. 53, No. 2, 2012, pp. 313-318. doi:10.1269/jrr.11107

[15] R. Pötter, P. George, J. C. Dimopoulos, M. Grimm, D. Berger, N. Nesvacil, et al., "Clinical Outcome of Protocol Based Image (MRI) Guided Adaptive Brachytherapy Combined with 3D Conformal Radiotherapy with or without Chemotherapy in Patients with Locally Advanced Cervical Cancer," Radiotherapy \& Oncology, Vol. 100, No. 1, 2011, pp. 116-123. doi:10.1016/j.radonc.2011.07.012

[16] J. C. Dimopoulos, S. Lang, C. Kirisits, E. F. Fidarova, D. Berger, P. Georg, et al., "Dose-Volume Histogram Parameters and Local Tumor Control in Magnetic Resonance Image-Guided Cervical Cancer Brachytherapy," International Journal of Radiation Oncology Biology Physics, Vol. 75, No. 1, 2009, pp. 56-63. doi:10.1016/j.ijrobp.2008.10.033

[17] P. Georg, S. Lang, J. C. Dimopoulos, W. Dörr, A. E. Sturdza, D. Berger, et al., "Dose-Volume Histogram Parameters and Late Side Effects in Magnetic Resonance Image-Guided Adaptive Cervical Cancer Brachytherapy," International Journal of Radiation Oncology Biology Physics, Vol. 79, No. 2, 2011, pp. 356-362. doi:10.1016/j.ijrobp.2009.11.002

[18] A. N. Viswanathan, J. Dimopoulos, C. Kirisits, D. Berger and R. Pötter, "Computed Tomography versus Magnetic Resonance Imaging-Based Contouring in Cervical Cancer Brachytherapy: Results of a Prospective Trial and Preliminary Guidelines for Standardized Contours," International Journal of Radiation Oncology Biology Physics, Vol. 68, No. 2, 2007, pp. 491-498. doi:10.1016/j.ijrobp.2006.12.021

[19] R. N. Eskander, D. Scanderbeg, C. C. Saenz, M. Brown and C. L. Yashar, "Comparison of Computed Tomography and Magnetic Resonance Imaging in Cervical Cancer Brachytherapy Target and Normal Tissue Contouring," International Journal of Gynecological Cancer, Vol. 20, No. 1, 2010, pp. 47-53. doi:10.1111/IGC.0b013e3181c4a627

[20] R. Krishnatry, F. D. Patel, P. Singh, S. C. Sharma, A. S. 
Oinam and A. K. Shukla, "CT or MRI for Image-based Brachytherapy in Cervical Cancer," Japanese Journal of Clinical Oncology, Vol. 42, No. 4, 2012, pp. 309-313. doi:10.1093/jico/hys010

[21] L. T. Tan, C. E. Coles, C. Hart and E. Tait, "Clinical Impact of Computed Tomography-Based Imaged-Guided Brachytherapy for Cervix Cancer Using the Tandem-Ring Applicator-The Addenbrooke's Experience," Clinical Oncology, Vol. 21, No. 3, 2009, pp. 175-182. doi:10.1016/j.clon.2008.12.001

[22] S. Van Dyk, K. Narayan, R. Fisher and D. Bernshaw, "Conformal Brachytherapy Planning for Cervical Cancer Using Transabdominal Ultrasound," International Journal of Radiation Oncology Biology Physics, Vol. 75, No. 1, 2009, pp. 64-70. doi:10.1016/j.ijrobp.2008.10.057

[23] U. Mahantshetty, N. Khanna, J. Swamidas, R. Engineer, M. H. Thakur, N. H. Merchant, et al., "Trans-Abdominal Ultrasound (US) and Magnetic Resonance Imaging (MRI) Correlation for Conformal Intracavitary Brachytherapy in Carcinoma of the Uterine Cervix," Radiotherapy \& Oncology, Vol. 102, No. 1, 2012, pp. 130-134. doi:10.1016/j.radonc.2011.08.001

[24] P. Trnkova, R. Poetter, D. Baltas, A. Karabis, E. Fidarova, J. Dimopoulos, et al., "New Inverse Planning Technology for Image-Guided Cervical Cancer Brachytherapy: Description and Evaluation within a Clinical Frame," $R a-$ diotherapy \& Oncology, Vol. 93, No. 2, 2009, pp. 331340. doi:10.1016/j.radonc.2009.10.004

[25] E. Chajon, I. Dumas, M. Touleimat, N. Magné, J. Coulot, R. Verstraet, et al., "Inverse-Planning Approach for 3-D MRI-Based Pulse-Dose Rate Intracavitary Brachytherapy in Cervix Cancer," International Journal of Radiation Oncology Biology Physics, Vol. 69, No. 3, 2007, pp. 955961. doi:10.1016/j.ijrobp.2007.07.2321

[26] D. H. Kim, A. Wang-Chesebro, V. Weinberg, J. Poulot, L. M. Chen, J. Speight, et al., "High-Dose Rate Brachytherapy Using Inverse Planning Simulated Annealing for Locoregionally Advanced Cervical Cancer: A Clinical Report with 2-yr Follow-Up," International Journal of Radiation Oncology Biology Physics, Vol. 75, No. 5, 2009, pp. 1329-1334. doi:10.1016/j.ijrobp.2009.01.002 